\title{
Bu open IFN production ability and healthy ageing: mixed model analysis of a 24 year longitudinal study in Japan
}

\author{
Kazuko Uno, ${ }^{1}$ Katsumi Yagi, ${ }^{1}$ Masayo Yoshimori, ${ }^{2}$ Mari Tanigawa, ${ }^{1}$ \\ Toshikazu Yoshikawa, ${ }^{1}$ Setsuya Fujita ${ }^{1}$
}

To cite: Uno K, Yagi K, Yoshimori $\mathrm{M}$, et al. IFN production ability and healthy ageing: mixed model analysis of a 24 year longitudinal study in Japan. BMJ Open 2013;3:e002113.

doi:10.1136/bmjopen-2012002113

- Prepublication history and additional material for this paper are available online. To view these files please visit the journal online (http://dx.doi.org/10.1136/ bmjopen-2012-002113).

Received 13 October 2012 Revised 17 December 2012 Accepted 18 December 2012

This final article is available for use under the terms of the Creative Commons Attribution Non-Commercial 2.0 Licence; see http://bmjopen.bmj.com

${ }^{1}$ Louis Pasteur Center for Medical Research, Sakyoku, Kyoto, Japan

${ }^{2}$ Division of Mathematical Science, Graduate School of Engineering Science, Osaka University, Toyonaka,

Osaka, Japan

Correspondence to: Dr Kazuko Uno;

kazukouno@louis-pasteur.or.jp

\section{ABSTRACT}

Objective: To track changes in interferon (IFN) production in healthy individuals to shed light on the effect these changes have on the course of healthy ageing.

Design: Study is based on data that were collected over 24 years from a cohort of individuals whose IFN- $\alpha$ production was quantified as a part of their annual routine health check-up.

Setting: All individuals in this study underwent regular health check-ups at Louis Pasteur Center for Medical Research.

Participants: 295 healthy individuals (159 males and 136 females) without a history of cancer, autoimmune diseases and hepatitis $C$ virus (HCV) whose IFN- $\alpha$ production was quantified more than five times within 24 years were selected. Finally, 29 males and 4 females whose IFN- $\alpha$ production was quantified more than 25 times were selected and their data were analysed using a mixed model.

Main outcome measures: HVJ stimulated IFN- $\alpha$ production was quantified. Healthy individual's periodical log transformed IFN- $\alpha$ values $(y)$ were plotted versus age $(x)$ and fitted to linear $(y=m x+n)$ and quadratic formula $\left(y=a x^{2}+b x+c\right)$ expressions to reveal changes in the IFN- $\alpha$ production in these healthy individuals.

Results: The linear expression showed that $\log ($ IFN- $\alpha)$ had a slight tendency to decline ( $3 \%$ over 10 years). However, the quadratic formula analysis showed the quadratic expression to be more positive than negative (a concave U-shaped pattern) which means that individuals' once declining IFN production recovered as they aged.

Conclusions: Although we observed a marginal decline in IFN- $\alpha$ production, we also observed that IFN production recovered even in individuals in their mid50s to early 60 s. These results combined with our previous cross-sectional studies of patients with various diseases suggest that in healthy individuals, the impairment of IFN production is triggered more by the onset of disease (notwithstanding the cause) rather than by ageing.

\section{INTRODUCTION}

There are many reports showing an age-related decline in immunity as humans

\section{ARTICLE SUMMARY}

Article focus

- To determine the changes in the interferon (IFN) production (a parameter of innate immunity) during the course of ageing through a 24-year longitudinal study.

- Creating an IFN production profile of healthy individuals to determine if these changes are a normal part of ageing or not.

Key messages

- It is widely believed that innate immunity continuously declines with age. This report partially agrees in that there is a decline in the IFN production; however the decline is relatively milder than is often believed.

- IFN production ability can increase after a decline even in healthy individuals who are in their mid50s to early 60 s.

- A severe decline in the IFN production is more a function of the onset of disease rather than ageing.

Strengths and limitations of this study

- There are no known reports that show the relationship between IFN production ability and ageing.

- Our results are based on a longitudinal study which is superior to the widely employed crosssectional study.

- The study population however was not of an ideal size.

get older. Ageing of the immune system leads to immunological susceptibility leading to infection, autoimmunity and malignancy, which may increase hospitalisation and mortality among the elders. ${ }^{1-3}$ It is well known that immune ageing is more severe in the acquired immune system than in the innate immune system. ${ }^{4-8}$ Some researchers have reported a significant decline in various aspects of the innate system such as decrease of phagocytosis, alteration of cellular migration, changes in innate immunity-related cell 
population and numbers, impaired cytokine production and phenotypic as well as functional changes. ${ }^{3}$ 9 10 Other researchers disagree however, and have reported only a marginal decline in innate immunity. ${ }^{11}$ On the contrary, it is widely agreed upon that the aspect of acquired immunity, such as the production of specific antibodies and naïve $\mathrm{T}$ and $\mathrm{B}$ cells number, decreases drastically with age. ${ }^{12} 13$

The human type I interferon (IFN) system performs basic host-defence functions, such as infection prevention and cancer immunological surveillance; ${ }^{14}$ it also promotes dendritic cell maturation thereby leading to specified T-helper cell differentiation. ${ }^{15-18}$ As a result of the multitude of roles it plays, the IFN system is important to the human innate and acquired immune systems. ${ }^{19}$ It comes as no surprise therefore that patients with type I IFN response deficiency (eg, stat 1, TYK2 gene mutation) or type I IFN production deficiency (eg, UNC93B1 gene mutation) are highly susceptible to infection. ${ }^{20} 21$

At our research centre, we established a unique IFN production test to analyse and explore the importance of the IFN system in humans. ${ }^{22}$ In order to achieve this, an immunological test in the form of IFN quantitation is conducted for all individuals (diseased and healthy) who undergo routine health check-ups at our research centre from 1987 to present. To shed light on the relationship between changes in IFN production and ageing, we took data from the aforementioned database, applied various selection criteria and performed a comprehensive data analysis.

Maintaining a high immune ability is important to ensure healthy ageing. Therefore, the rational of this research was to observe long-term IFN production in a cohort of individuals as this is a good parameter to gain insights into the immune ability and the health status of individuals. If, through this and other research, it is determined that a reduction occurs even in healthy ageing then no medical intervention is required for individuals whose IFN production decreases with age. However, if it is ascertained that a decreased IFN production is not a function of healthy ageing, then the cause and an appropriate treatment or life style changes need to be undertaken.

In a previous paper focusing on patients with various diseases, we demonstrated that irrespective of their age, patients with lung cancer, diabetes, myelodysplasteic syndrome, advanced immunoglobulin A (IgA) nephritis, HIV and HCV have impaired IFN- $\alpha$ production. ${ }^{22-26}$ In addition, based on records from a 17-year follow-up study (data are a part of the 24-year database), a comparison of age-matched healthy individuals and HCV patients revealed that impairment of IFN- $\alpha$ production was likely linked to an increase in the cancer risk. ${ }^{25}$ Furthermore, we also showed that IFN- $\alpha$ production decline was not only evident in lung cancer and HCV patients but also evident in patients who developed diabetes mellitus. ${ }^{26}$ Therefore, based on these previous results, we have understood that in spite of the cause (infectious disease, cancer, metabolic disease and nephritis), many kinds of diseases impair IFN- $\alpha$ production and that IFN dysfunction leads to a higher risk of infectious diseases and cancer development.

Longitudinal studies are logistically and financially difficult, especially those involving long-term observation of immune response. As a result, only a few have been reported including the Swedish OCTO, NOVAimmune. $^{12} 27$ Baltimore and Hisayama studies, all of which seem to continue to yield exciting data. ${ }^{28-30}$ Many researchers have analysed healthy ageing using crosssectional studies involving a comparison of young and elderly individuals; this method however can yield biased data. Elements that affect healthy ageing such as difference in genetics, natural environment, eating habits and other lifestyle choices are not considered in cross-sectional studies. As a result, cross-sectional studies offer only a limited understanding of healthy ageing. To overcome this limitation we performed a longitudinal study which offers superior results. In this study, our aim was to determine the immune/age relationship in healthy individuals. We believe that results from prior cross-sectional studies are insufficient to prove the wellknown belief that ageing negatively affects immune ability. For these reasons, we opted to support our theory with data obtained retrospectively from our 24-year database. In this paper, we will shed light on the changes in IFN production in the course of healthy ageing and consider the effects of disease development on the IFN system.

\section{METHODS}

\section{Study population}

Medical records were obtained for 2387 individuals who underwent health check-ups at Louis Pasteur Center for Medical Research from January 1987 to December 2010. Individuals' records included data on their IFN- $\alpha$ production values in addition to their results from routine laboratory blood tests. These individuals consisting of healthy individuals and those with various diseases had on average two visits per year per person with a combined total of 9480 recorded visits. Blood samples were taken from individuals after obtaining written informed consent. All individuals are long-term club members who support the research of Louis Pasteur Center for Medical Research.

\section{Statistical analysis}

A primary analysis was conducted using the IFN- $\alpha$ production values for 2387 individuals. From this cohort, 295 individuals were selected; each satisfied the following conditions: (1) their IFN- $\alpha$ production was quantified more than five times and (2) they had no history of cancer, autoimmune or chronic infectious diseases. Their periodical $\log$ transformed IFN- $\alpha$ values $(y)$ were plotted versus age $(\mathrm{x})$ and then fitted to linear $(\mathrm{y}=\mathrm{mx}+\mathrm{n})$ and 


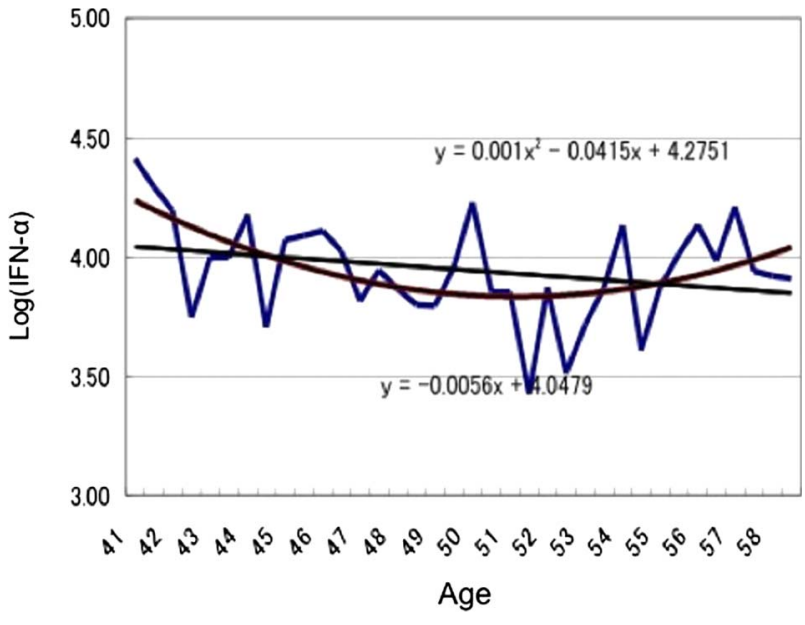

Figure 1 Longitudinal interferon (IFN) $\alpha$ production data of one individual whose pattern is typical of the majority of the sample population. individuals' periodical log transformed IFN- $\alpha$ values $(y)$ were plotted versus age (x) and fitted to (1) a linear expression $(y=m x+n)$ and (2) quadratic formula $\left(y=a x^{2}+b x+c\right)$.

quadratic formula $\left(y=\mathrm{ax}^{2}+\mathrm{bx}+\mathrm{c}\right)$ expressions as shown in figure 1. Regression analyses were performed using the SAS statistical software package V.8.2 (SAS Institute Inc, North Carolina, USA).

The results of the primary analysis revealed that when IFN- $\alpha$ production was measured more than 25 times, the resulting values showed an overall tendency to stabilise as shown in figure 2. A subzero tendency in the linear expression and a plus tendency in the quadratic expression clearly emerged from the data points. Therefore, from the group of 295 healthy individuals, we selected 33 (designated as long-term monitored (LT) individuals) whose IFN- $\alpha$ production was quantified more than 25 times. We then analysed the data using a mixed model analysis with the statistical software R. A diagram of the selection process is shown in figure 3 .
Measurement of IFN production in whole blood

The method for quantifying IFN- $\alpha$ production in whole human blood has been described previously. ${ }^{22}$ Briefly, heparinised peripheral blood samples were used within $8 \mathrm{~h}$ of IFN induction. Samples were cultured with Sendai virus (HVJ) and incubated at $37^{\circ} \mathrm{C}$ for $20 \mathrm{~h}$. Supernatants were harvested and IFN- $\alpha$ activity in the supernatants was determined by bioassay (using FL cells and Sindbis virus) following which we determined the titer value. IFN titre was standardised to WHO international reference IFN- $\alpha$ MRC 69/19B, 95/568 and we later used Japanese standard interferon- $\alpha$ (J501) from 2005. These were standardised using international standard 95/574 over the period of the study.

\section{RESULTS}

\section{Primary analysis}

The log-transformed value of each individual's periodical IFN- $\alpha$ values (y) was plotted versus age (x) and fitted to two quadratic formula expressions $(y=m x+n$ and $\left.y=a x^{2}+b x+c\right)$, as shown in figure 1. These individuals' IFN- $\alpha$ production was quantified for $9.2 \pm 0.2$ years and $13.4 \pm 0.5$ times $($ mean $\pm \mathrm{SE})$.

As shown in figure $2 \mathrm{~A}, \mathrm{~B}$, the coefficient $m$ values of the linear expression showed that more individuals displayed declining IFN production when IFN was quantified more than 25 times. The coefficient of each IFN measurement frequency level showed a negative value, suggesting a declining trend.

On the contrary, the results of the quadratic formula analysis showed that the population mean of the coefficient $a$ of the quadratic expression was more positive than negative (figure $2 \mathrm{C}, \mathrm{D}$ ). More frequent quantification of individuals' IFN production resulted in a clear concave U shape, which means that individuals' once declining IFN production recovered as they aged. There
Figure 2 Distribution of a coefficient value. (A) Value of the coefficient $m$ was plotted for individuals whose interferon (IFN) $\alpha$ was quantified more than five times. (B) Close-up the distribution of the values found in the red oval of $2 \mathrm{a}$. The oval consists of the values for individuals whose IFN was quantified over 25 times.

(C) Each value of the coefficient a was plotted more than five times.

(D) Close-up the distribution of the values found in the red oval of 2c. The oval consists of IFN quantification that was performed over 25 times. (a)

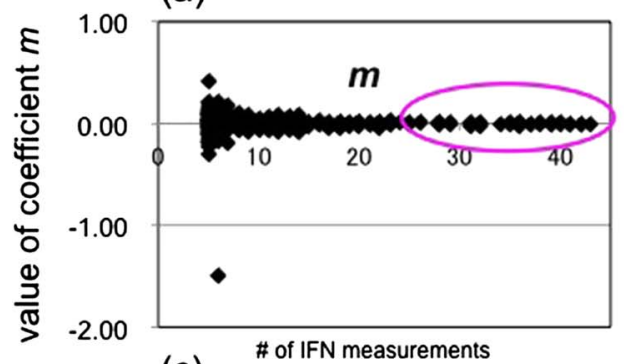

(c)

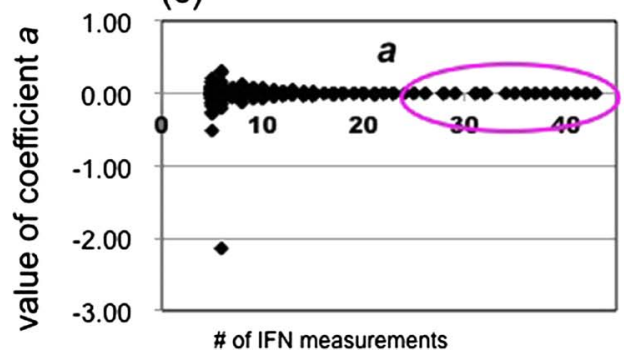

(b)

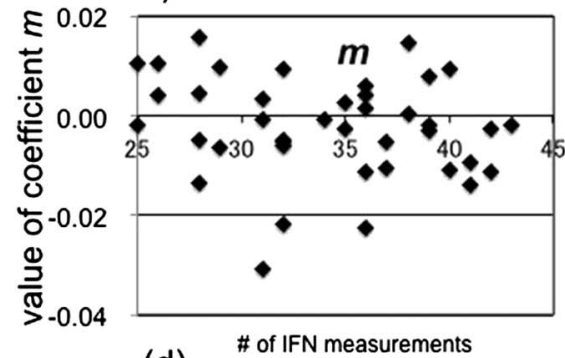

(d)

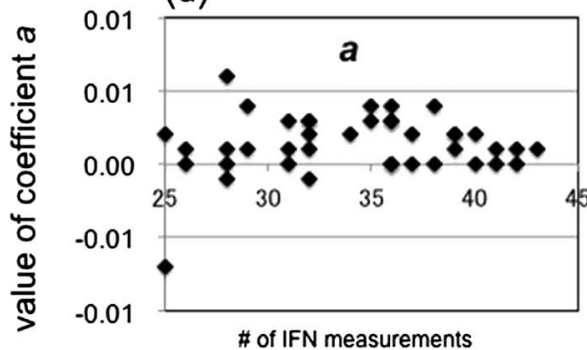


Selection method for subjects

\begin{tabular}{|c|}
\hline $\begin{array}{l}\text { Data base of a total of } 9480 \text { recorded visits logged by } 2387 \text { subjects whose IFN- } \alpha \\
\text { production and health check test were compiled over } 24 \text { years (from } 1987 \text { to 2010) } \\
\text { at Louis Pasteur Center for Medical Research. }\end{array}$ \\
\hline \\
\hline Healthy subjects \\
\hline $\begin{array}{l}\text { Subjects without a history of cancer, autoimmune diseases and HCV were } \\
\text { selected from the above group. }\end{array}$ \\
\hline \\
\hline $\begin{array}{l}\text { From the above group, subjects whose IFN- } \alpha \text { production was quantified more than } \\
5 \text { times were selected: } n=295 \text { (Male: } 159 \text {, Female:136) }\end{array}$ \\
\hline \\
\hline $\begin{array}{l}\text { Finally, subjects whose IFN- } \alpha \text { production was quantified more than } 25 \text { times } \\
\text { (regularly once or twice a year) were selected, and analyzed using mixed model: } \\
\text { n=33 (Male:29, Female:4) }\end{array}$ \\
\hline
\end{tabular}

Figure 3 Selection process for individuals.

were no observable differences between male and female IFN production tendencies (data not shown).

\section{Mixed model analysis}

The results of the primary analysis revealed that after 25 measurements, IFN production showed an overall tendency to stabilise. We therefore selected 33 LT healthy individuals whose IFN production was measured more than 25 times for the mixed model analysis (male: $n=29$, female: $n=4)$. These 33 LT healthy individuals had their IFN production quantified once or twice a year consistently for more than 15 years (mean $\pm \mathrm{SD} ; 17.84 \pm 0.37$ years, $34.09 \pm 0.87$ times) (see online supplementary figure $\mathrm{S} 1$ ). As shown in table 1A, the results of the linear expression fitting showed that IFN production in LT healthy individuals showed a slight but statistically significant, tendency to decline $(p=0.045$, table 1$)$. The population mean of the coefficient of the linear expression value was -0.003 , indicating that the $\log (\mathrm{IFN}-\alpha$ value $)$ declined $3 \%$ over 10 years $(10-25 \%$ of actual value).

With regard to the quadratic formula analysis of LT healthy individuals, results showed that the population mean of the coefficient $a$ of the quadratic expression was positive (a concave U-shaped pattern), revealing that LT individuals' once declining IFN production recovered as they aged (this was statistically significant, $\mathrm{p}=0.0107$, table 1). The age distribution of the lowest IFN- $\alpha$ production values falls between 55 and 65 years (figure 4).

\section{DISCUSSION}

In our 24-year longitudinal study, a mixed model analysis of the linear expression revealed that $\log (\mathrm{IFN}-\alpha)$, which is the body's basic immune-defence ability, marginally declined $3 \%$ over 10 years even in healthy individuals. This means for example that an original IFN- $\alpha$ value of 10000 or $5802 \mathrm{IU} / \mathrm{ml}$ declined to 7585 and $4473 \mathrm{IU} / \mathrm{ml}$, respectively, over 10 years. This decline in IFN production was approximately half of what we anticipated. This may be because the individuals enrolled were determined healthy based on blood tests that ruled out the possibility of cancer and other chronic diseases. Surprisingly, the results of the quadratic formula analysis showed that the population mean of the coefficient $a$ of the quadratic expression was positive (a concave U-shaped pattern), which means that individuals' once declining IFN production recovered as they aged. This strongly suggests that IFN- $\alpha$ production can increase after a decline even in individuals who are in their mid-50s or early 60 s.

There are no known studies about the effect of ageing on the IFN system, however one report has suggested that the number of plasmacytoid cells which are the main producers of IFN- $\alpha$ is significantly low in the aged. ${ }^{31}$ On the contrary, another report has stated that the number of myeloid dendritic cells (DCs) declined in the aged but plasmacytoid DC in the peripheral blood showed no significant decline in elderly individuals. ${ }^{10}$ Unlike innate immunity where there are opposing views, there is a general consensus among researchers that there is a severe decline in acquired immunity, such as naïve $\mathrm{T}$ cell number and naïve $\mathrm{B}$ cell number, antibody response and vaccination efficacy. ${ }^{46}$ In innate immunity a severe decline is appropriately half to two-thirds of original function, while for acquired immunity, a severe decline is generally as much as a nine-tenth drop in the immune function.

As was previously stated, the results of the quadratic formula analysis of LT healthy individuals showed that the population mean of the coefficient $a$ of the quadratic expression was positive (a concave U-shaped pattern), which means that LT individuals' once declining IFN production recovered as they aged. This finding is very significant since it is widely believed that immune ageing can only be reversed if the disease that caused the decline in immune ability is successfully treated. In our previous paper, we demonstrated that recovery from lung tuberculosis or successful anti-HIV therapy increased the IFN- $\alpha$ production ${ }^{22}$ (partially unpublished data). Although it has been reported that several cytokines show increased production in the aged these tend to be

\begin{tabular}{|c|c|c|c|c|c|}
\hline Fixed effects & Value & SE & Degree of freedom & t Value & p Value \\
\hline \multicolumn{6}{|c|}{ A: The results of linear expression analysis } \\
\hline \multicolumn{6}{|c|}{ B: The results of quadratic formula analysis } \\
\hline$x^{2}: a^{2}$ & a: 0.00026 & 0.000102 & 1090 & 2.55 & 0.0107 \\
\hline
\end{tabular}




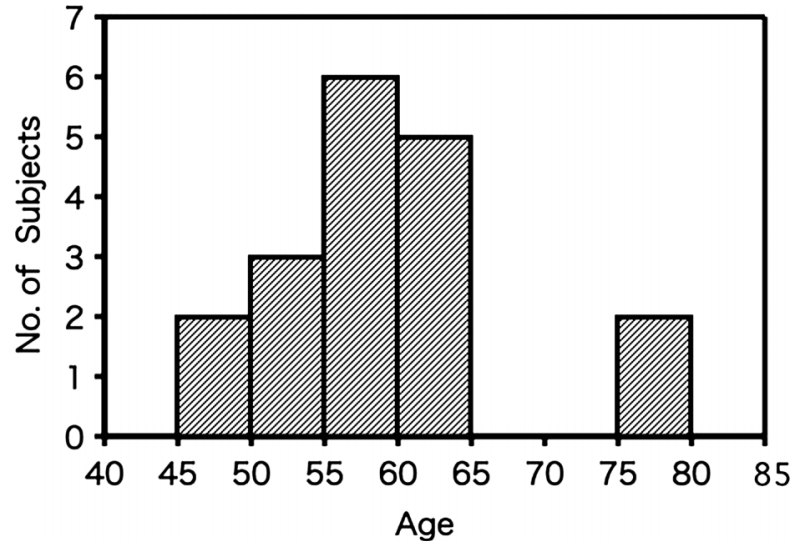

Figure 4 Distribution of individuals' lowest interferon (IFN) production age. Of the 33 individuals, 18 were selected whose lowest IFN production occurred within the observation period of this study.

immunosuppressive or inflammatory cytokines ${ }^{32} 33$ Thus, while many responses in the innate immune system diminish with age, there is substantial evidence of age-associated hyper-reactivity of innate immunity as well.

We observed that the lowest IFN- $\alpha$ production occurred when individuals were between 55 and 65 years old. Why is IFN- $\alpha$ production lowest at this stage? The vast majority of the individuals in this study are middle class business executives or self-employed individuals who are particularly health conscious and therefore visit our Institute twice a year for health check-ups. In Japan, the average retirement age falls between 60 and 65 years and we surmise that this important milestone may be the cause of increased stress and anxiety resulting in a drop in the IFN- $\alpha$ production. The Japanese Ohsaki study which comprised of about 50000 adults aged 40 years or older residing in Ohsaki City, Miyagi Prefecture, demonstrated that psychological distress levels (using the K6, a six-item instrument that assesses non-specific psychological distress developed by Kessler) showed a clear U-shaped curve, with lowest stress levels occurring at age $60-69$ years. ${ }^{34}$ Our study mirrors this result; lowest IFN- $\alpha$ production occurred between the ages of 55 and 65 and a recovery, which we have linked to reduce stress levels began within this period. While most reports showing improved health after retirement are dated, a recent report has suggested that a reduction in prior job strains after retirement may result in improved health especially for some individuals who had stressful jobs ${ }^{35}$ Furthermore, a study using the French GAZEL occupational cohort also concluded that retirement was associated with a substantial reduction in mental and physical fatigue as well as depressive symptoms. ${ }^{25} 37$

This is the first known study using a longitudinal approach to demonstrate that IFN- $\alpha$ production ability can recover in middle-aged persons. We find these results encouraging, since they suggest that if an average healthy person experienced a decline in the IFN- $\alpha$ production, recovery is possible with a change in the lifestyle that includes a significant reduction in stress.

Our previously reported results showed that patients with lung cancer, diabetes, myelodysplasteic syndrome, advanced IgA nephritis, HIV and HCV irrespective of the cause of the disease showed impaired IFN- $\alpha$ production. ${ }^{22}{ }^{25}$ In addition, we showed that IFN- $\alpha$ production declined gradually, not only with the development of $\mathrm{HCV}$, but also with an increase in the plasma blood sugar level. ${ }^{25} 26$ These findings combined with our present results support our conclusion that severe IFN dysfunction is not derived from ageing, but from the onset of various types of diseases (infectious disease, cancer, metabolic disease and nephritis). Based on these findings, we propose periodic assessment and quantification of IFN- $\alpha$ production to discover changes in the IFN system, in order to determine if individuals are ageing healthily, susceptible to disease or are in the early stage of the onset of a disease. Using this information, treatment or changes in the lifestyle can be recommended which may lead to a recovery in the IFN- $\alpha$ production and an improvement in an individual's health.

\section{Strengths and limitations}

One of the main strengths of this report lies in the fact that it is a 24-year longitudinal study versus a crosssectional study. The limitations of cross-sectional surveys are that they provide data only for a specific or single point in time and are unable therefore to reveal any meaningful change in the sample from year to year. Since a study about ageing needs to track the changes in a population over time, a cross-sectional survey would not accurately reflect the actual status. On the contrary, longitudinal studies are designed to trace changes in a given population over a period of time and therefore trends can be observed by comparing the data obtained in separate years. Our longitudinal study allowed us to repeatedly measure the same population over 24 years thereby giving more reliable data.

Although at 33, our sample is somewhat small, the same results could be seen in our sample of 295 individuals whose IFN was quantified more than five times (mean 9.2 \pm 0.2 years and mean $13.4 \pm 0.5$ times). Another limitation is that although we presumed that preretirement stress was the reason for individuals' lowest IFN- $\alpha$ production occurring between 55 and 65 years old, we have no hard data to show a causal relationship. We therefore relied on the findings of prior reports, and our knowledge that retirees in that age group are usually senior managers with responsibilities that entail a great deal of stress. This may partly explain why IFN- $\alpha$ production recovered when individuals were between the ages of 60 and 65, which reflect the period after individuals had retired.

\section{Future direction/further research}

In this report, only healthy individuals were analysed. We also possess data for cancer individuals who had 
treatments following their IFN test or who were diagnosed with cancer during the period that their IFN was being quantified. We believe that further analysis is likely to reveal the IFN- $\alpha$ production pattern that differs in healthy individuals and individuals with diseases.

Acknowledgements We thank all the individuals who made this study possible through their long-term trust in our Institute and their decision to use us as their premier choice for medical services. We extend our gratitude to the research and administrative staff of the Louis Pasteur Center for Medical Research for their assistance with this project. A special thanks extended to Dr T Kishida (who passed away in 2006), and Dr J Setoguchi for being thorough in the clinical follow-up during the initial phase of this research. We are grateful to Ms L. Russell for the substantive editing of this manuscript.

Contributors KU participated in the design and coordination of this study from 1987, as well as the acquisition of data, statistical analysis, data interpretation, drafting and critical revision of the paper. KY and MY completed statistical analysis of the data. MT collected and verified the clinical information of the study participants, and TY and SF provided data analysis support. All authors approved the final version of the paper.

Funding A part of this work was supported by Grants-in-Aid for Scientific Research from the Ministry of Education, Science, Sports and Culture of Japan (No.17606005, No. 16201041), the Japan Health Foundation (2010) and the Shimizu Foundation Research Grant (2005). All authors are independent from all the above funding.

Competing interests None.

Ethics approval Approval was obtained from the Louis Pasteur Center for Medical Research Ethical Committees (LPC.8). All participants gave written informed consent.

Provenance and peer review Not commissioned; externally peer reviewed.

Data sharing statement There are no additional data available.

\section{REFERENCES}

1. Larbi A, Franceschi C, Mazzatti D, et al. Aging of the immune system as a prognostic factor for human longevity. Physiology (Bethesda) 2008;23:64-74.

2. Weinberger B, Herndler-Brandstetter D, Schwanninger A, et al. Biology of immune responses to vaccines in elderly persons. Clin Infect Dis 2008;46:1078-84.

3. Ponnappan S, Ponnappan U. Aging and immune function: molecular mechanisms to interventions. Antioxid Redox Signal 2011;14:1551-85.

4. Hirokawa K, Utsuyama M, Kasai M, et al. Aging and immunity. Acta Pathol Jpn 1992:42:537-48.

5. Fulop T, Larbi A, Wikby A, et al. Dysregulation of T-cell function in the elderly: scientific basis and clinical implications. Drug Aging 2005;22:589-603.

6. Herndler-Brandstetter D, Cioca DP, Grubeck-Loebenstein B. Immunizations in the elderly: do they live up to their promise? Wien Med Wochenschr (1946) 2006;156:130-41.

7. Kovaiou RD, Grubeck-Loebenstein B. Age-associated changes within CD4+ T cells. Immunol Lett 2006;107:8-14.

8. Derhovanessian E, Larbi A, Pawelec G. Biomarkers of human immunosenescence: impact of Cytomegalovirus infection. Curr Opin Immunol 2009:21:440-5.

9. Franceschi C, Bonafe M. Centenarians as a model for healthy aging Biochem Soc Transact 2003;31:457-61.

10. Bella S Della, Bierti L, Presicce $P$, et al. Peripheral blood dendritic cells and monocytes are differently regulated in the elderly. Clin Immunol 2007;122:220-8.

11. Alonso-Fernandez P, Puerto M, Mate I, et al. Neutrophils of centenarians show function levels similar to those of young adults. $J$ Am Geriatr Soc 2008;56:2244-51.

12. Wikby A, Maxson P, Olsson J, et al. Changes in CD8 and CD4 lymphocyte subsets, $T$ cell proliferation responses and non-survival in the very old: the Swedish longitudinal OCTO-immune study. Mech Ageing Dev 1998;102:187-98.
13. Pawelec $\mathrm{G}$, Derhovanessian $\mathrm{E}$. Role of $\mathrm{CMV}$ in immune senescence. Virus Res 2011;157:175-9.

14. Baron S. The biological significance of the interferon system. Arch Intern Med 1970;126:84-93.

15. Le Bon A, Schiavoni G, D’Agostino G, et al. Type i interferons potently enhance humoral immunity and can promote isotype switching by stimulating dendritic cells in vivo. Immunity 2001;14:461-70.

16. Santini SM, Di Pucchio T, Lapenta C, et al. The natural alliance between type I interferon and dendritic cells and its role in linking innate and adaptive immunity. $J$ Interferon Cytokine Res 2002;22:1071-80.

17. Gabriele L, Borghi P, Rozera C, et al. IFN-alpha promotes the rapid differentiation of monocytes from patients with chronic myeloid leukemia into activated dendritic cells tuned to undergo full maturation after LPS treatment. Blood 2004;103:980-7.

18. Rizza P, Moretti F, Belardelli F. Recent advances on the immunomodulatory effects of IFN-alpha: implications for cancer immunotherapy and autoimmunity. Autoimmunity 2010;43:204-9.

19. Belardelli F, Ferrantini M, Proietti E, et al. Interferon-alpha in tumor immunity and immunotherapy. Cytokine Growth Factor Rev 2002;13:119-34.

20. Jouanguy E, Altare F, Lamhamedi-Cherradi S, et al. Infections in IFNGR-1-deficient children. J Interferon Cytokine Res 1997;17:583-7.

21. Jouanguy E, Zhang SY, Chapgier A, et al. Human primary immunodeficiencies of type I interferons. Biochimie 2007;89:878-83.

22. Uno K, Nakano K, Maruo N, et al. Determination of interferon-alpha-producing capacity in whole blood cultures from patients with various diseases and from healthy persons. J Interferon Cytokine Res 1996;16:911-18.

23. Shirakawa K, Muso E, Nogaki F, et al. Correlation between the severity of clinicopathological parameters and whole blood interferon-alpha production capacity in active phase IgA nephropathy patients. Nephron 2002;90:24-30.

24. Uno K, Suginoshita Y, Kakimi K, et al. Impairment of IFN-alpha production capacity in patients with hepatitis $C$ virus and the risk of the development of hepatocellular carcinoma. World J Gastroenterol 2005;11:7330-4.

25. Uno K, Hirosaki M, Kakimi K, et al. Impaired IFN-alpha production and the risk of cancer development. $J$ Interferon Cytokine Res 2007;27:1013-17.

26. Tominaga M, Uno K, Yagi K, et al. Association between capacity of interferon-alpha production and metabolic parameters. J Interferon Cytokine Res 2010;30:451-4.

27. Nilsson B-O, Ernerudh J, Johansson B, et al. Morbidity does not influence the T-cell immune risk phenotype in the elderly: findings in the Swedish NONA Immune Study using sample selection protocols. Mech Ageing Dev 2003;124:469-76.

28. O'Brien RJ, Resnick SM, Zonderman AB, et al. Neuropathologic studies of the Baltimore Longitudinal Study of Aging (BLSA). $J$ Alzheimers Dis: JAD 2009;18:665-75.

29. Doi Y, Ninomiya T, Hata J, et al. Impact of glucose tolerance status on development of ischemic stroke and coronary heart disease in a general Japanese population: the Hisayama study. Stroke 2010;41:203-9.

30. lida M, Ikeda F, Ninomiya T, et al. White blood cell count and risk of gastric cancer incidence in a general Japanese population: the Hisayama study. Am J Epidemiol 2012;175:504-10.

31. Sridharan A, Esposo M, Kaushal K, et al. Age-associated impaired plasmacytoid dendritic cell functions lead to decreased CD4 and CD8 T cell immunity. Age (Dordrecht, Netherlands) 2011;33:363-76.

32. Caruso C, Lio D, Cavallone L, et al. Aging, longevity, inflammation, and cancer. Ann N Y Acad Sci 2004;1028:1-13.

33. Franceschi $\mathrm{C}$, Bonafe $\mathrm{M}$, Valensin $\mathrm{S}$, et al. Inflamm-aging. An evolutionary perspective on immunosenescence. Ann NY Acad Sci 2000;908:244-54

34. Kuriyama S, Nakaya N, Ohmori-Matsuda K, et al. The Ohsak Cohort 2006 Study: design of study and profile of participants at baseline. J Epidemiol/Jpn Epidemiol Assoc 2010;20:253-8.

35. Ekerdt DJ, Bosse R, LoCastro JS. Claims that retirement improves health. J Gerontol 1983;38:231-6.

36. Sjosten $\mathrm{N}$, Nabi $\mathrm{H}$, Westerlund $\mathrm{H}$, et al. Influence of retirement and work stress on headache prevalence: a longitudinal modelling study from the GAZEL Cohort Study. Cephalalgia 2011;31:696-705.

37. Westerlund $\mathrm{H}$, Vahtera J, Ferrie JE, et al. Effect of retirement on major chronic conditions and fatigue: French GAZEL occupational cohort study. BMJ 2010;341:c6149. 